\title{
RACSAM
}

Rev. R. Acad. Cien. Serie A. Mat.

VOL. 102 (2), 2008, pp. 211-220

Análisis Matemático / Mathematical Analysis

\section{Mixed intersections of non quasi-analytic classes}

\author{
Jean Schmets and Manuel Valdivia
}

\begin{abstract}
Given two semi-regular matrices $\mathfrak{M}$ and $\mathfrak{M}^{\prime}$ and two open subsets $\Omega$ and $\Omega^{\prime}$ [resp. two compact subsets $K$ and $\left.K^{\prime}\right]$ of $\mathbb{R}^{r}$ and $\mathbb{R}^{s}$ respectively, we introduce the spaces $\mathcal{E}_{\left(\mathfrak{M} \times \mathfrak{M}^{\prime}\right)}\left(\Omega \times \Omega^{\prime}\right)$ and $\mathcal{D}_{\left(\mathfrak{M} \times \mathfrak{M}^{\prime}\right)}\left(\Omega \times \Omega^{\prime}\right)$ [resp. $\mathcal{D}_{\left(\mathfrak{M} \times \mathfrak{M}^{\prime}\right)}\left(K \times K^{\prime}\right)$ ]. In this paper we study their locally convex properties and the structure of their elements. This leads in [10] to tensor product representations of these spaces and to some kernel theorems.
\end{abstract}

Intersecciones mixtas de clases no casi-analíticas

Resumen. Dadas dos matrices semi-regulares $\mathfrak{M}$ y $\mathfrak{M}^{\prime}$ y dos subconjuntos abiertos $\Omega$ y $\Omega^{\prime}$ [respectivamente dos subconjuntos compactos $K$ y $\left.K^{\prime}\right]$ de $\mathbb{R}^{r}$ y $\mathbb{R}^{s}$ respectivamente, introducimos los espa$\operatorname{cios} \mathcal{E}_{\left(\mathfrak{M} \times \mathfrak{M}^{\prime}\right)}\left(\Omega \times \Omega^{\prime}\right)$ y $\mathcal{D}_{\left(\mathfrak{M} \times \mathfrak{M}^{\prime}\right)}\left(\Omega \times \Omega^{\prime}\right)$ [respectivamente $\mathcal{D}_{\left(\mathfrak{M} \times \mathfrak{M}^{\prime}\right)}\left(K \times K^{\prime}\right)$ ]. En este artículo estudiamos sus propiedades localmente convexas y la estructura de sus elementos, lo que nos ha permitido en [10] obtener representaciones de estos espacios en productos tensoriales y algunos teoremas de núcleos.

\section{Introduction}

Intersections of non quasi-analytic classes have first been investigated by Chaumat and Chollet in [3] in the case $M_{j, p}=M_{p}^{a_{j}}$ where $\left(M_{p}\right)_{p \in \mathbb{N}_{0}}$ is a sequence with moderate growth and $\left(a_{j}\right)_{j \in \mathbb{N}}$ a sequence of positive numbers strictly decreasing to 0 . They obtained a Whitney extension theorem, a Łojasiewicz theorem on regular situation, some theorem of division and preparation and a Whitney spectral theorem.

Later on Beaugendre studied extensively such intersections in [1] and [2] when the numbers $M_{j, p}$ are defined by means of a convex and increasing function $\Phi$ on $\left[0,+\infty\right.$ [ such that $\lim _{t \rightarrow \infty} \Phi(t) / t=\infty$. In particular he obtained extension results for Whitney jets and an explicit continuous linear map for Whitney jets.

We considered such intersections for general matrices $\mathfrak{M}=\left(M_{j, p}\right)_{j \in \mathbb{N}, p \in \mathbb{N}_{0}}$ and obtained analytic and holomorphic extensions of Whitney jets in [6] and an explicit continuous linear extension map for Whitney jets in [7].

In [9] we investigated locally convex properties of these intersections.

In [8] we introduced mixed non quasi-analytic classes, i.e. given two increasing normalized and non quasi-analytic sequences $\boldsymbol{M}$ and $\boldsymbol{M}^{\prime}$ of positive numbers as well as two open subsets $\Omega$ and $\Omega^{\prime}$ [resp. two compact subsets $K$ and $\left.K^{\prime}\right]$ of $\mathbb{R}^{r}$ and $\mathbb{R}^{s}$ respectively, we consider the spaces $\mathcal{E}^{\left(M, M^{\prime}\right)}\left(\Omega \times \Omega^{\prime}\right)$ and $\mathcal{D}^{\left(M, M^{\prime}\right)}\left(\Omega \times \Omega^{\prime}\right)$ [resp. $\mathcal{D}^{\left(M, M^{\prime}\right)}\left(K \times K^{\prime}\right)$ ]. We studied some of their locally convex properties,

Presentado por / Submitted by Manuel López Pellicer.

Recibido / Received: 14 de mayo de 2008. Aceptado / Accepted: 4 de junio de 2008 .

Palabras clave / Keywords: ultradifferentiable functions, Beurling classes, nuclearity.

Mathematics Subject Classifications: 46A11, 46A32, 46E10, 46F05.

(c) 2008 Real Academia de Ciencias, España. 
approximation and denseness properties and the structure of their elements. This lead to tensor product representations and kernel theorems.

In this paper we investigate the case of intersections of mixed non quasi-analytic classes: locally convex properties and structure of their elements. We present tensor product characterizations of them and develop some kernel theorems in [10].

\section{Notations}

All functions we consider are complex valued and all vector spaces are $\mathbb{C}$-vector spaces. The euclidean norm of $x \in \mathbb{R}^{n}$ is denoted $|x|$. If $f$ is a function defined on $A \subset \mathbb{R}^{n}$, then we set $\|f\|_{A}:=\sup _{x \in A}|f(x)|$.

If $E$ is a Hausdorff locally convex topological vector space (in short: a locally convex space), $E^{\prime}$ designates its topological dual endowed with the strong $\beta\left(E^{\prime}, E\right)$ topology. We refer to [4] and [5] for properties of the locally convex spaces.

Whenever $\boldsymbol{m}$ is a sequence $\left(m_{p}\right)_{p \in \mathbb{N}_{0}}$ of real numbers, the notation $\boldsymbol{M}$ designates as usual the sequence $\left(M_{p}\right)_{p \in \mathbb{N}_{0}}$ where $M_{p}=m_{0} \ldots m_{p}$ for every $p \in \mathbb{N}_{0}$. Such a sequence is

(a) normalized if $m_{0}=1$ and $m_{p} \geq 1$ for every $p \in \mathbb{N}$;

(b) non quasi-analytic if $\sum_{p=0}^{\infty} 1 / m_{p}<\infty$.

A semi-regular matrix $\mathfrak{m}$ is a matrix of the type

$$
\mathfrak{m}=\left(m_{j, p}\right)_{j \in \mathbb{N}, p \in \mathbb{N}_{0}}
$$

such that, for every $j \in \mathbb{N}$, the sequence $\boldsymbol{m}_{j}=\left(m_{j, p}\right)_{p \in \mathbb{N}_{0}}$ is normalized, increasing, non quasi-analytic and such that

(a) $m_{j, p} \geq m_{j+1, p}$ for every $p \in \mathbb{N}_{0}$,

(b) $\lim _{p \rightarrow \infty} m_{j+1, p} / m_{j, p}=0$.

Then, of course, $\boldsymbol{M}_{j}$ designates the sequence $\left(M_{j, p}\right)_{p \in \mathbb{N}_{0}}$ for every $j \in \mathbb{N}$ and $\mathfrak{M}$ the matrix $\left(M_{j, p}\right)_{j \in \mathbb{N}, p \in \mathbb{N}_{0}}$ and, for every open subset $\Omega$ and compact subset $K$ of $\mathbb{R}^{n}$ as in [8], one can introduce

a) the (FS)-space $\mathcal{E}_{(\mathfrak{M})}(\Omega)$ as the projective limit of the spaces $\mathcal{E}^{\left(M_{j}\right)}(\Omega)$, i.e. the space of the $\mathcal{C}^{\infty}$ functions $f$ on $\Omega$ such that

$$
\|f\|_{H, h, j}:=\sup _{\alpha \in \mathbb{N}_{0}^{n}} \frac{\left\|\mathrm{D}^{\alpha} f\right\|_{H}}{h^{|\alpha|} M_{j,|\alpha|}}<\infty
$$

for every compact subset $H$ of $\Omega, h>0$ and $j \in \mathbb{N}$, endowed with the fundamental system of semi-norms $\left\{\|\cdot\|_{H, h, j}: H \Subset \Omega, h>0, j \in \mathbb{N}\right\}$;

b) the $(\mathrm{FS})$-space $\mathcal{D}_{(\mathfrak{M})}(K)$ as the topological subspace of $\mathcal{E}_{(\mathfrak{M})}\left(\mathbb{R}^{n}\right)$ of the elements having their support contained in $K$;

c) the (LFS)-space $\mathcal{D}_{(\mathfrak{M})}(\Omega)$ as the inductive limit of the spaces $\mathcal{D}_{(\mathfrak{M})}(H)$ where $H$ runs through the family of the compact subsets of $\Omega$.

From now on, unless otherwise stated,

(a) $r$ and $s$ are positive integers;

(b) $\Omega$ and $\Omega^{\prime}$ are non empty open subsets of $\mathbb{R}^{r}$ and $\mathbb{R}^{s}$ respectively;

(c) $\mathfrak{m}=\left(m_{j, p}\right)_{j \in \mathbb{N}, p \in \mathbb{N}_{0}}$ and $\mathfrak{m}^{\prime}=\left(m_{j, p}^{\prime}\right)_{j \in \mathbb{N}, p \in \mathbb{N}_{0}}$ are semi-regular matrices.

Then $\mathcal{E}_{\left(\mathfrak{M} \times \mathfrak{M}^{\prime}\right)}\left(\Omega \times \Omega^{\prime}\right)$ is the vector space of the $\mathcal{C}^{\infty}$-functions $f$ on $\Omega \times \Omega^{\prime}$ such that, for every compact subset $K$ of $\Omega$ and $K^{\prime}$ of $\Omega^{\prime}, h>0$ and $j \in \mathbb{N}$,

$$
\|f\|_{K \times K^{\prime}, h, j}=\sup _{(\alpha, \beta) \in \mathbb{N}_{0}^{r} \times \mathbb{N}_{0}^{s}} \frac{\left\|\mathrm{D}^{(\alpha, \beta)} f\right\|_{K \times K^{\prime}}}{h^{|\alpha|+|\beta|} M_{j,|\alpha|} M_{j,|\beta|}^{\prime}}<\infty,
$$

endowed with the fundamental system of the semi-norms $\|\cdot\|_{K \times K^{\prime}, h, j}$ where $K$ (resp. $K^{\prime}$ ) runs through the non void compact subsets of $\Omega$ (resp. $\Omega^{\prime}$ ), $h>0$ and $j \in \mathbb{N}$. As it coincides with the projective limit of the (FS)-spaces $\mathcal{E}^{\left(\boldsymbol{M}_{j}, \boldsymbol{M}_{j}^{\prime}\right)}\left(\Omega \times \Omega^{\prime}\right)$ (cf. [8]), it is a (FS)-space. 
Given a compact subset $H$ of $\mathbb{R}^{r} \times \mathbb{R}^{s}$, we designate by $\mathcal{D}_{\left(\mathfrak{M} \times \mathfrak{M}^{\prime}\right)}(H)$ the topological subspace of $\mathcal{D}_{\left(\mathfrak{M} \times \mathfrak{M}^{\prime}\right)}\left(\mathbb{R}^{r} \times \mathbb{R}^{s}\right)$, the elements of which have their support contained in $H$. It clearly is a (FS)-space.

Finally $\mathcal{D}_{\left(\mathfrak{M} \times \mathfrak{M}^{\prime}\right)}\left(\Omega \times \Omega^{\prime}\right)$ designates of course the inductive limit of the Fréchet spaces $\mathcal{D}_{\left(\mathfrak{M} \times \mathfrak{M}^{\prime}\right)}(H)$ where $H$ runs through the non void compact subsets of $\Omega \times \Omega^{\prime}$. It is a (LFS)-space. In particular we consider it as the inductive limit of the spaces $\mathcal{D}_{\left(\mathfrak{M} \times \mathfrak{M}^{\prime}\right)}\left(K_{n} \times K_{n}^{\prime}\right)$ where $\left(K_{n}\right)_{n \in \mathbb{N}}$ and $\left(K_{n}^{\prime}\right)_{n \in \mathbb{N}}$ are compact exhaustions of $\Omega$ and $\Omega^{\prime}$ respectively, such that $K_{n} \subset K_{n+1}^{\circ}$ and $K_{n}^{\prime} \subset K_{n+1}^{\prime \circ}$ for every $n \in \mathbb{N}$.

\section{First properties}

Proposition 1 The bilinear map

$$
\Lambda: \mathcal{E}_{\left(\mathfrak{M} \times \mathfrak{M}^{\prime}\right)}\left(\Omega \times \Omega^{\prime}\right)^{2} \rightarrow \mathcal{E}_{\left(\mathfrak{M} \times \mathfrak{M}^{\prime}\right)}\left(\Omega \times \Omega^{\prime}\right) ; \quad(f, g) \mapsto f g
$$

is well defined and continuous.

PROOF. Given sequences $\left(f_{m}\right)_{m \in \mathbb{N}}$ and $\left(g_{m}\right)_{m \in \mathbb{N}}$ converging to 0 in $\mathcal{E}_{\left(\mathfrak{M} \times \mathfrak{M}^{\prime}\right)}\left(\Omega \times \Omega^{\prime}\right)$, Proposition 3.1 of [8] tells us that, for every $j \in \mathbb{N}$, the sequence $\left(f_{m} g_{m}\right)_{m \in \mathbb{N}}$ converges to 0 in $\mathcal{E}^{\left(\boldsymbol{M}_{j}, \boldsymbol{M}_{j}^{\prime}\right)}\left(\Omega \times \Omega^{\prime}\right)$.

Definition 1 By $\mathcal{E}^{(p ! q !)}\left(\mathbb{R}^{r} \times \mathbb{R}^{s}\right)$, we designate the space of the $\mathcal{C}^{\infty}$-functions $f$ on $\mathbb{R}^{r} \times \mathbb{R}^{s}$ such that, for any $h>0$ and any non void compact subsets $K$ of $\mathbb{R}^{r}$ and $K^{\prime}$ of $\mathbb{R}^{s}$,

$$
|f|_{K \times K^{\prime}, h}=\sup _{(\alpha, \beta) \in \mathbb{N}_{0}^{r} \times \mathbb{N}_{0}^{s}} \frac{\left\|D^{(\alpha, \beta)} f\right\|_{K \times K^{\prime}}}{h^{|\alpha|+|\beta|} \alpha ! \beta !}<\infty
$$

endowed with the system $\left\{|\cdot|_{K \times K^{\prime}, h}: K \Subset \mathbb{R}^{r}, K^{\prime} \Subset \mathbb{R}^{s}, h>0\right\}$ of semi-norms. It clearly is a Fréchet space.

We also denote by $\mathcal{H}\left(\mathbb{C}^{n}\right)$ the Fréchet space of the holomorphic functions on $\mathbb{C}^{n}$ endowed with the topology of uniform convergence on the compact subsets of $\mathbb{C}^{n}$. Classical holomorphy arguments easily provide that the restriction map

$$
\Gamma: \mathcal{H}\left(\mathbb{C}^{r+s}\right) \rightarrow \mathcal{E}^{(p ! q !)}\left(\mathbb{R}^{r} \times \mathbb{R}^{s}\right) ;\left.\quad f \mapsto f\right|_{\mathbb{R}^{r+s}}
$$

is a well defined isomorphism.

Proposition 2 The vector space $\mathcal{E}^{(p ! q !)}\left(\mathbb{R}^{r} \times \mathbb{R}^{s}\right)$ is a vector subspace of $\mathcal{E}_{\left(\mathfrak{M} \times \mathfrak{M}^{\prime}\right)}\left(\mathbb{R}^{r} \times \mathbb{R}^{s}\right)$ and the corresponding canonical injection is continuous.

PROOF. For every $j \in \mathbb{N},\left(m_{j, p}\right)_{p \in \mathbb{N}_{0}}$ and $\left(m_{j, p}^{\prime}\right)_{p \in \mathbb{N}_{0}}$ are increasing sequences of positive numbers which are non quasi-analytic. This implies $\lim _{p \rightarrow \infty} p / m_{j, p}=0$ and $\lim _{p \rightarrow \infty} p / m_{j, p}^{\prime}=0$. Therefore there is $p_{0} \in \mathbb{N}$ such that $p \leq m_{j, p}$ and $p \leq m_{j, p}^{\prime}$ for every integer $p \geq p_{0}$ hence a constant $B_{j}>0$ such that

$$
p ! \leq B_{j} M_{j, p} \quad \text { and } \quad p ! \leq B_{j} M_{j, p}^{\prime}, \quad \forall p \in \mathbb{N}_{0} .
$$

The conclusion is then immediate since this leads to

$$
\|f\|_{K \times K^{\prime}, h, j} \leq B_{j}^{2}|f|_{K \times K^{\prime}, h}, \quad \forall f \in \mathcal{E}^{(p ! q !)}\left(\mathbb{R}^{r} \times \mathbb{R}^{s}\right),
$$

for all $h>0, j \in \mathbb{N}$ and compact subsets $K$ of $\mathbb{R}^{r}$ and $K^{\prime}$ of $\mathbb{R}^{s}$.

By Proposition 5.3 of [9], we know that, for every compact subsets $H$ and $K$ of $\Omega$ such that $H \subset K^{\circ}$ [resp. $H^{\prime}$ and $K^{\prime}$ of $\Omega^{\prime}$ such that $H^{\prime} \subset K^{\prime \circ}$ ], there is $f \in \mathcal{D}_{(\mathfrak{M})}(K)$ identically 1 on a neighbourhood of $H$ [resp. $g \in \mathcal{D}_{\left(\mathfrak{M}^{\prime}\right)}\left(K^{\prime}\right)$ identically 1 on a neighbourhood of $H^{\prime}$ ], so it is a standard matter to obtain the following results. 
Lemma 1 a) For every compact subsets $H$ and $K$ of $\Omega \times \Omega^{\prime}$ such that $K \subset H^{\circ}$, there is a positive function $\varphi \in \mathcal{D}_{\left(\mathfrak{M} \times \mathfrak{M}^{\prime}\right)}(H)$ which is identically 1 on a neighbourhood of $K$.

b) If $\left\{\Omega_{j}: j \in J\right\}$ is a finite open cover of the compact subset $K$ of $\Omega \times \Omega^{\prime}$, then there are positive functions $\varphi_{j} \in \mathcal{D}_{\left(\mathfrak{M} \times \mathfrak{M}^{\prime}\right)}\left(\Omega \times \Omega^{\prime}\right)$ such that $\operatorname{supp}\left(\varphi_{j}\right) \subset \Omega_{j}$ for every $j \in J$ and $\sum_{j \in J} \varphi_{j}$ is identically 1 on a neighbourhood of $K$.

c) For every open cover $\mathcal{O}$ of $\Omega \times \Omega^{\prime}$, there is a $\mathcal{D}_{\left(\mathfrak{M} \times \mathfrak{M}^{\prime}\right)}\left(\Omega \times \Omega^{\prime}\right)$-partition of unity subordinate to $\mathcal{O}$.

Proposition 3 The set $\mathcal{D}_{\left(\mathfrak{M} \times \mathfrak{M}^{\prime}\right)}\left(\Omega \times \Omega^{\prime}\right)$ is a dense vector subspace of $\mathcal{E}_{\left(\mathfrak{M} \times \mathfrak{M}^{\prime}\right)}\left(\Omega \times \Omega^{\prime}\right)$.

Definition 2 A 0-open set of the $\mathfrak{M} \times \mathfrak{M}^{\prime}$-distribution $u$ on $\Omega \times \Omega^{\prime}$ [i.e. a continuous linear functional on $\left.\mathcal{D}_{\left(\mathfrak{M} \times \mathfrak{M}^{\prime}\right)}\left(\Omega \times \Omega^{\prime}\right)\right]$ is an open subset $\omega$ of $\Omega \times \Omega^{\prime}$ such that $\langle u, \varphi\rangle=0$ for every $\varphi \in \mathcal{D}_{\left(\mathfrak{M} \times \mathfrak{M}^{\prime}\right)}(\omega)$.

Proposition 4 Every union of 0-open sets of a $\mathfrak{M} \times \mathfrak{M}^{\prime}$-distribution u on $\Omega \times \Omega^{\prime}$ is a 0 -open set of $u$.

Definition 3 The support $\operatorname{supp}(u)$ of the $\mathfrak{M} \times \mathfrak{M}^{\prime}$-distribution $u$ on $\Omega \times \Omega^{\prime}$ is the complement in $\Omega \times \Omega^{\prime}$ of the 0-open set of u, i.e. the union of all the 0-open sets of $u$.

Proposition 5 A $\mathfrak{M} \times \mathfrak{M}^{\prime}$-distribution has a compact support if and only if it has a continuous linear extension on $\mathcal{E}_{\left(\mathfrak{M} \times \mathfrak{M}^{\prime}\right)}\left(\Omega \times \Omega^{\prime}\right)$.

\section{Approximation}

Notation 1 Given $b \in \mathbb{R}^{n}$ and a function $f$ defined on $\mathbb{R}^{n}, \tau_{b} f$ designates the function defined on $\mathbb{R}^{n}$ by $\tau_{b} f()=.f(.-b)$.

Proposition 6 For every $b \in \mathbb{R}^{r} \times \mathbb{R}^{s}$, the map $\tau_{b}$ is a well defined continuous linear map from the space $\mathcal{E}_{\left(\mathfrak{M} \times \mathfrak{M}^{\prime}\right)}\left(\mathbb{R}^{r} \times \mathbb{R}^{s}\right)$ into itself.

Moreover we have $\lim _{|b| \rightarrow 0} \tau_{b} f=f$ for every $f \in \mathcal{E}_{\left(\mathfrak{M} \times \mathfrak{M}^{\prime}\right)}\left(\mathbb{R}^{r} \times \mathbb{R}^{s}\right)$.

PROOF. This is a direct consequence of Proposition 7.3 of [8] stating that, for every $j \in \mathbb{N}$, the analogous result is valid for $\mathcal{E}^{\left(\boldsymbol{M}_{j}, \boldsymbol{M}_{j}^{\prime}\right)}\left(\mathbb{R}^{r} \times \mathbb{R}^{s}\right)$.

Notation 2 For every $m \in \mathbb{N}, \psi_{m}$ designates the function defined on $\mathbb{R}^{r} \times \mathbb{R}^{s}$ by

$$
\psi_{m}(u, v)=m^{(r+s)} \pi^{-(r+s) / 2} e^{-m^{2}|u|^{2}} e^{-m^{2}|v|^{2}}, \quad \forall(u, v) \in \mathbb{R}^{r} \times \mathbb{R}^{s} .
$$

Proposition 7 For every $m \in \mathbb{N}$ and $f \in \mathcal{D}_{\left(\mathfrak{M} \times \mathfrak{M}^{\prime}\right)}\left(\mathbb{R}^{r} \times \mathbb{R}^{s}\right)$, the function $f \star \psi_{m}$ has a holomorphic extension on $\mathbb{C}^{r+s}$ and therefore belongs to $\mathcal{E}^{(p ! q !)}\left(\mathbb{R}^{r} \times \mathbb{R}^{s}\right)$.

Proposition 8 For every element $f$ of the space $\mathcal{D}_{\left(\mathfrak{M} \times \mathfrak{M}^{\prime}\right)}\left(\mathbb{R}^{r} \times \mathbb{R}^{s}\right)$, the sequence $\left(f \star \psi_{m}\right)_{m \in \mathbb{N}}$ converges to $f$ in $\mathcal{E}_{\left(\mathfrak{M} \times \mathfrak{M}^{\prime}\right)}\left(\mathbb{R}^{r} \times \mathbb{R}^{s}\right)$.

Proof. Clearly $\left(f \star \psi_{m}\right)_{m \in \mathbb{N}}$ is a sequence of $\mathcal{E}^{(p ! q !)}\left(\mathbb{R}^{r} \times \mathbb{R}^{s}\right)$ hence of $\mathcal{E}_{\left(\mathfrak{M} \times \mathfrak{M}^{\prime}\right)}\left(\mathbb{R} \times \mathbb{R}^{s}\right)$ by Proposition 2.

The conclusion then follows directly from Proposition 5.2 of [8] saying that, for every $j \in \mathbb{N}$, the sequences $\left(f \star \psi_{m}\right)_{m \in \mathbb{N}}$ converges to $f$ in $\mathcal{E}^{\left(\boldsymbol{M}_{j}, \boldsymbol{M}_{j}^{\prime}\right)}\left(\mathbb{R}^{r} \times \mathbb{R}^{s}\right)$.

Proposition 9 The set of the restrictions of the polynomials to $\Omega \times \Omega^{\prime}$ is a dense vector subspace of $\mathcal{E}_{\left(\mathfrak{M} \times \mathfrak{M}^{\prime}\right)}\left(\Omega \times \Omega^{\prime}\right)$.

PRoof. This follows directly from Proposition 5.3 of [8] saying that, for every $j \in \mathbb{N}$, the set of the restrictions of the polynomials to $\Omega \times \Omega^{\prime}$ is dense in $\mathcal{E}^{\left(\boldsymbol{M}_{j}, \boldsymbol{M}_{j}^{\prime}\right)}\left(\Omega \times \Omega^{\prime}\right)$. 
Proposition 10 The vector space $\mathcal{D}_{(\mathfrak{M})}(\Omega) \otimes \mathcal{D}_{\left(\mathfrak{M}^{\prime}\right)}\left(\Omega^{\prime}\right)$ is dense in

(a) $\mathcal{E}_{\left(\mathfrak{M} \times \mathfrak{M}^{\prime}\right)}\left(\Omega \times \Omega^{\prime}\right)$;

(b) $\mathcal{D}_{\left(\mathfrak{M} \times \mathfrak{M}^{\prime}\right)}\left(\Omega \times \Omega^{\prime}\right)$.

PROoF. (a) By Corollary 9, it suffices to establish that the set of the restrictions to $\Omega \times \Omega^{\prime}$ of the polynomials on $\mathbb{R}^{r} \times \mathbb{R}^{s}$ is contained in the closure of $\mathcal{D}_{(\mathfrak{M})}(\Omega) \otimes \mathcal{D}_{\left(\mathfrak{M}^{\prime}\right)}\left(\Omega^{\prime}\right)$ in $\mathcal{E}_{\left(\mathfrak{M} \times \mathfrak{M}^{\prime}\right)}\left(\Omega \times \Omega^{\prime}\right)$.

This is a standard matter. We choose fundamental sequences $\left(K_{n}\right)_{n \in \mathbb{N}}$ and $\left(K_{n}^{\prime}\right)_{n \in \mathbb{N}}$ of compact subsets of $\Omega$ and $\Omega^{\prime}$ respectively, such that $K_{n} \subset K_{n+1}^{\circ}$ and $K_{n}^{\prime} \subset K_{n+1}^{\prime \circ}$ for every $n \in \mathbb{N}$. Next, for every $n \in \mathbb{N}$, we let $f_{n}$ (resp. $g_{n}$ ) be an element of $\mathcal{D}_{(\mathfrak{M})}\left(K_{n+1}\right)$ (resp. of $\mathcal{D}_{\left(\mathfrak{M}^{\prime}\right)}\left(K_{n+1}^{\prime}\right)$ identically 1 on a neighbourhood of $K_{n}$ (resp. $K_{n}^{\prime}$ ). Then, for every polynomial $P$ on $\mathbb{R}^{r} \times \mathbb{R}^{s}$, we have

$$
\left(f_{n} \otimes g_{n}\right) \cdot P \in \mathcal{D}_{(\mathfrak{M})}(\Omega) \otimes \mathcal{D}_{\left(\mathfrak{M}^{\prime}\right)}\left(\Omega^{\prime}\right), \quad \forall n \in \mathbb{N},
$$

and the sequence $\left(\left(f_{n} \otimes g_{n}\right) \cdot P\right)_{n \in \mathbb{N}}$ converges to $P$ in $\mathcal{E}_{\left(\mathfrak{M} \times \mathfrak{M}^{\prime}\right)}\left(\Omega \times \Omega^{\prime}\right)$.

(b) Let $h$ be an element of $\mathcal{D}_{\left(\mathfrak{M} \times \mathfrak{M}^{\prime}\right)}\left(\Omega \times \Omega^{\prime}\right)$ and $\left(P_{n}\right)_{n \in \mathbb{N}}$ be a sequence of polynomials converging to $h$ in $\mathcal{E}_{\left(\mathfrak{M} \times \mathfrak{M}^{\prime}\right)}\left(\Omega \times \Omega^{\prime}\right)$. Let moreover $K, H$ (resp. $\left.K^{\prime}, H^{\prime}\right)$ be compact subsets of $\Omega$ (resp. $\Omega^{\prime}$ ) such that $\operatorname{supp}(h) \subset K \times K^{\prime}$ and $K \times K^{\prime} \subset H^{\circ} \times H^{\prime \circ}$. Then we choose $f \in \mathcal{D}_{(\mathfrak{M})}(H)$ and $g \in \mathcal{D}_{\left(\mathfrak{M}^{\prime}\right)}\left(H^{\prime}\right)$ identically 1 on a neighbourhood of $K$ and $K^{\prime}$ respectively. Then the sequence $\left((f \otimes g) . P_{n}\right)_{n \in \mathbb{N}}$ of $\mathcal{D}_{\left(\mathfrak{M} \times \mathfrak{M}^{\prime}\right)}\left(\Omega \times \Omega^{\prime}\right)$ converges to $(f \otimes g) . h=h$ in $\mathcal{E}_{\left(\mathfrak{M} \times \mathfrak{M}^{\prime}\right)}\left(\Omega \times \Omega^{\prime}\right)$. As we have $\operatorname{supp}(f \otimes g) \cdot P_{n} \subset H \times H^{\prime}$ for every $m \in \mathbb{N}$, we conclude at once.

Definition 4 A subset $B$ of $\mathbb{R}^{n}$ has the local displacement property if every $x \in B$ has a neighbourhood $W$ such that, for every $\varepsilon>0$, there is $a \in \mathbb{R}^{n}$ such that $|a| \leq \varepsilon$ and $a+B \cap W \subset B^{\circ}$.

If $B_{1}, \ldots, B_{q}$ are closed balls in $\mathbb{R}^{n}$ in finite number and such that $B_{j} \cap B_{k} \neq \emptyset$ implies $B_{j}^{\circ} \cap B_{k}^{\circ} \neq \emptyset$, one can check that their union has the local displacement property. Moreover if the compact subsets $K$ of $\mathbb{R}^{r}$ and $K^{\prime}$ of $\mathbb{R}^{s}$ have the local displacement property, it is clear that $K \times K^{\prime}$ also has this property.

Therefore, from now on, we agree that the exhaustions $\left(K_{n}\right)_{n \in \mathbb{N}}$ of $\Omega$ and $\left(K_{n}^{\prime}\right)_{n \in \mathbb{N}}$ of $\Omega^{\prime}$ consist of compact sets having the local displacement property and such that $K_{n} \subset K_{n+1}^{\circ}$ and $K_{n}^{\prime} \subset K_{n+1}^{\prime \circ}$ for every $n \in \mathbb{N}$.

Proposition 11 If the compact subsets $K$ of $\mathbb{R}^{r}$ and $K^{\prime}$ of $\mathbb{R}^{s}$ have the local displacement property, then $\mathcal{D}_{(\mathfrak{M})}(K) \otimes \mathcal{D}_{\left(\mathfrak{M}^{\prime}\right)}\left(K^{\prime}\right)$ is a dense vector subspace of $\mathcal{D}_{\left(\mathfrak{M} \times \mathfrak{M}^{\prime}\right)}\left(K \times K^{\prime}\right)$.

PROOF. One has just to adapt the proof of Proposition 8.1 of [8] which provides a similar result for each of the spaces $\mathcal{D}^{\left(M_{j}, M_{j}^{\prime}\right)}\left(K \times K^{\prime}\right)$.

\section{Structure of the elements of $\mathcal{E}_{\left(\mathfrak{M} \times \mathfrak{M}^{\prime}\right)}\left(\Omega \times \Omega^{\prime}\right)$ and $\mathcal{D}_{\left(\mathfrak{M} \times \mathfrak{M}^{\prime}\right)}\left(\Omega \times \Omega^{\prime}\right)$}

Definition 5 Let us say that $\mathfrak{m}^{\prime}$ (or equivalently $\mathfrak{M}^{\prime}$ ) is regular if, for every $j \in \mathbb{N}$, there are constants $A(j), H(j)>1$ such that

$$
M_{j+1, p+1}^{\prime} \leq A(j) H(j)^{p} M_{j, p}^{\prime}, \quad \forall p \in \mathbb{N} .
$$

Proposition 12 a) For every $f \in \mathcal{E}_{\left(\mathfrak{M} \times \mathfrak{M}^{\prime}\right)}\left(\Omega \times \Omega^{\prime}\right)$ and $y \in \Omega^{\prime}$, the function $f(\cdot, y)$ belongs to $\mathcal{E}_{(\mathfrak{M})}(\Omega)$.

b) If $\mathfrak{M}^{\prime}$ is regular, then, for every $\beta \in \mathbb{N}^{s}, D^{(0, \beta)}$ is a continuous linear map from $\mathcal{E}_{\left(\mathfrak{M} \times \mathfrak{M}^{\prime}\right)}\left(\Omega \times \Omega^{\prime}\right)$ into itself.

PROOF. a) is straightforward: $f(\cdot, y)$ is a $\mathcal{C}^{\infty}$-function on $\Omega$ and, for every continuous semi-norm $\|\cdot\|_{K, h, j}$ on $\mathcal{E}_{(\mathfrak{M})}(\Omega),\|\cdot\|_{K \times\{y\}, h, j}$ is a continuous semi-norm on $\mathcal{E}_{\left(\mathfrak{M} \times \mathfrak{M}^{\prime}\right)}\left(\Omega \times \Omega^{\prime}\right)$ such that

$$
\|f(\cdot, y)\|_{K, h, j}=\sup _{\alpha \in \mathbb{N}_{0}^{r}} \frac{\left\|\mathrm{D}^{(\alpha, 0)} f\right\|_{K \times\{y\}}}{h^{|\alpha|} M_{j,|\alpha|} M_{j, 0}^{\prime}} \leq\|f\|_{K \times\{y\}, h, j} .
$$


b) Let $\|\cdot\|_{K \times K^{\prime}, h, j}$ be any continuous semi-norm on $\mathcal{E}_{\left(\mathfrak{M} \times \mathfrak{M}^{\prime}\right)}\left(\Omega \times \Omega^{\prime}\right)$. As $\mathfrak{M}^{\prime}$ is regular, it is a direct matter to get constants $A, H>1$ such that

$$
M_{j+|\beta|, p+|\beta|}^{\prime} \leq A H^{|\beta| p} M_{j, p}^{\prime}, \quad \forall p \in \mathbb{N}_{0} .
$$

Therefore we get

$$
\left\|\mathrm{D}^{(0, \beta)} f\right\|_{K \times K^{\prime}, h, j}=\sup _{(\alpha, \gamma) \in \mathbb{N}_{0}^{r} \times \mathbb{N}_{0}^{s}} \frac{\left\|\mathrm{D}^{(\alpha, \beta+\gamma)} f\right\|_{K \times K^{\prime}}}{h^{|\alpha|+|\gamma|} M_{j,|\alpha|} M_{j,|\gamma|}^{\prime}} \leq A h^{|\beta|}\|f\|_{K \times K^{\prime}, h / H|\beta|, j+|\beta|}
$$

for every $f \in \mathcal{E}_{\left(\mathfrak{M} \times \mathfrak{M}^{\prime}\right)}\left(\Omega \times \Omega^{\prime}\right)$ and we conclude at once.

Proposition 13 If $\mathfrak{M}^{\prime}$ is regular, then, for every $f \in \mathcal{E}_{\left(\mathfrak{M} \times \mathfrak{M}^{\prime}\right)}\left(\Omega \times \Omega^{\prime}\right)$, the function

$$
g: \Omega^{\prime} \rightarrow \mathcal{E}_{(\mathfrak{M})}(\Omega) ; \quad y \mapsto f(\cdot, y)
$$

is $\mathcal{C}^{\infty}$ and, for every $\beta \in \mathbb{N}_{0}^{s}$, one has $D^{\beta} g(y)=D^{(0, \beta)} f(\cdot, y)$ for every $y \in \Omega^{\prime}$.

PROOF. Up to considering the real and imaginary parts of $f$ separately, we may suppose $f$ real.

We first prove that, for every $\beta \in \mathbb{N}_{0}^{s}$, the function

$$
g_{\beta}: \Omega^{\prime} \rightarrow \mathcal{E}_{(\mathfrak{M})}(\Omega) ; \quad y \mapsto \mathrm{D}^{(0, \beta)} f(\cdot, y)
$$

is continuous. Consider any continuous semi-norm $\|\cdot\|_{K, h, j}$ on $\mathcal{E}_{(\mathfrak{M})}(\Omega)$ and point $y$ of $\Omega^{\prime}$. There is $d>0$ such that $\left\{v \in \mathbb{R}^{s}:|y-v| \leq d\right\} \subset \Omega^{\prime}$ and, for every $v \in \mathbb{R}^{s}$ such that $|y-v| \leq d$, the limited Taylor formula leads to

$$
\begin{aligned}
\| \mathrm{D}^{(0, \beta)} f(\cdot, y) & -\mathrm{D}^{(0, \beta)} f(\cdot, v) \|_{K, h, j} \\
& \leq \sup _{\alpha \in \mathbb{N}_{0}^{r}} \sup _{x \in K} \sup _{\theta \in[0,1]} \sum_{k=1}^{s}\left|y_{k}-v_{k}\right| \frac{\left|\mathrm{D}^{\left(\alpha, \beta+\epsilon_{k}\right)} f(x, y+\theta(v-y))\right|}{h^{|\alpha|} M_{j,|\alpha|}} \\
& \leq s|y-v| h^{|\beta|+1} M_{j,|\beta|+1}^{\prime}\|f\|_{K \times\left\{z \in \mathbb{R}^{s}:|y-z| \leq d\right\}, h, j}
\end{aligned}
$$

and the conclusion of this part of the proof is immediate.

To conclude, it is now sufficient to establish that, for every $\beta \in \mathbb{N}_{0}^{s}$ such that $|\beta|=1$, we have $\mathrm{D}^{\beta} g(y)=$ $\mathrm{D}^{(0, \beta)} f(\cdot, y)$ for every $y \in \Omega^{\prime}$. Let us consider for instance $\beta=\epsilon_{1}$ and $y \in \Omega^{\prime}$; we need to prove that

$$
\lim _{k \rightarrow 0}\left\|\frac{g\left(y+k \epsilon_{1}\right)-g(y)}{k}-\mathrm{D}^{\left(0, \epsilon_{1}\right)} f(\cdot, y)\right\|_{K, h, j}=0
$$

for any continuous semi-norm $\|\cdot\|_{K, h, j}$ on $\mathcal{E}_{(\mathfrak{M})}(\Omega)$. There is $d>0$ such that $K^{\prime}:=\left\{v \in \mathbb{R}^{s}:|y-v| \leq\right.$ $d\} \subset \Omega^{\prime}$. Given $\varepsilon>0$, we first choose $m \in \mathbb{N}$ such that

$$
2^{-m} h M_{j, 1}^{\prime}\left\|\mathrm{D}^{\left(0, \epsilon_{1}\right)} f\right\|_{K \times K^{\prime}, h / 2, j} \leq \frac{\varepsilon}{2} .
$$

For any $k \in \mathbb{R}$ such that $0<|k| \leq d$, acting as in the first part of the proof leads to

$$
\begin{aligned}
& \left\|\frac{g\left(y+k \epsilon_{1}\right)-g(y)}{k}-\mathrm{D}^{\left(0, \epsilon_{1}\right)} f(\cdot, y)\right\|_{K, h, j} \\
& \quad \leq \sup _{|\alpha|<m} \sup _{\substack{x \in K \\
z \in K^{\prime}}} \frac{\left|\mathrm{D}^{\left(\alpha, \epsilon_{1}\right)} f(x, z)-\mathrm{D}^{\left(\alpha, \epsilon_{1}\right)} f(x, y)\right|}{h^{|\alpha|} M_{j,|\alpha|}}+2 \sup _{|\alpha| \geq m} \sup _{\substack{x \in K \\
z \in K^{\prime}}} \frac{\left|\mathrm{D}^{\left(\alpha, \epsilon_{1}\right)} f(x, z)\right|}{h^{|\alpha|} M_{j,|\alpha|}} .
\end{aligned}
$$


Now on one hand we note that we have

$$
\sup _{|\alpha| \geq m} \sup _{\substack{x \in K \\ z \in K^{\prime}}} \frac{\left|\mathrm{D}^{\left(\alpha, \epsilon_{1}\right)} f(x, z)\right|}{h^{|\alpha|} M_{j,|\alpha|}} \leq 2^{-m-1} h M_{j, 1}^{\prime}\left\|\mathrm{D}^{\left(0, \epsilon_{1}\right)} f\right\|_{K \times K^{\prime}, h / 2, j}
$$

hence the second term of the second member in the previous inequality is $\leq \varepsilon / 2$. On the other hand, the set of functions $\left\{\mathrm{D}^{\left(\alpha, \epsilon_{1}\right)} f: \alpha \in \mathbb{N}_{0}^{r},|\alpha|<m\right\}$ is uniformly equicontinuous on $K \times K^{\prime}$; this provides the existence of $\delta \in] 0, d]$ such that

$$
\sup _{|\alpha|<m} \sup _{\substack{x \in K \\|y-z| \leq \delta}} \frac{\left|\mathrm{D}^{\left(\alpha, \epsilon_{1}\right)} f(x, z)-\mathrm{D}^{\left(\alpha, \epsilon_{1}\right)} f(x, y)\right|}{h^{|\alpha|} M_{j,|\alpha|}} \leq \frac{\varepsilon}{2} .
$$

Hence the conclusion.

Proposition 14 Let $\mathfrak{M}^{\prime}$ be regular. If u belongs to $\mathcal{E}_{(\mathfrak{M})}(\Omega)^{\prime}$,

(a) the function $\langle u, f(\cdot, y)\rangle$ belongs to $\mathcal{E}_{\left(\mathfrak{M}^{\prime}\right)}\left(\Omega^{\prime}\right)$ for every $f \in \mathcal{E}_{\left(\mathfrak{M} \times \mathfrak{M}^{\prime}\right)}\left(\Omega \times \Omega^{\prime}\right)$. Moreover one has $D^{\beta}\langle u, f(\cdot, y)\rangle=\left\langle u, D^{(0, \beta)} f(\cdot, y)\right\rangle$ for every $\beta \in \mathbb{N}_{0}^{s}$.

(b) the linear map

$$
\Lambda: \mathcal{E}_{\left(\mathfrak{M} \times \mathfrak{M}^{\prime}\right)}\left(\Omega \times \Omega^{\prime}\right) \rightarrow \mathcal{E}_{\left(\mathfrak{M}^{\prime}\right)}\left(\Omega^{\prime}\right) ; \quad f \mapsto \Lambda f(y)=\langle u, f(\cdot, y)\rangle
$$

is continuous. More precisely, if u verifies $|\langle u, \cdot\rangle| \leq C\|\cdot\|_{K_{0}, h_{0}, j_{0}}$ on $\mathcal{E}_{(\mathfrak{M})}(\Omega)$, then we have

$$
\|\Lambda \cdot\|_{K^{\prime}, h, j} \leq C\|\cdot\|_{K_{0} \times K^{\prime}, h, j}
$$

on $\mathcal{E}_{\left(\mathfrak{M} \times \mathfrak{M}^{\prime}\right)}\left(\Omega \times \Omega^{\prime}\right)$ for every $\left.h \in\right] 0, h_{0}\left[\right.$ and $j \geq j_{0}$.

PROOF. From the previous two propositions, we obtain that $\langle u, f(\cdot, y)\rangle$ is a $\mathcal{C}^{\infty}$-function on $\Omega^{\prime}$ such that $\mathrm{D}^{\beta}\langle u, f(\cdot, y)\rangle=\left\langle u, \mathrm{D}^{(0, \beta)} f(\cdot, y)\right\rangle$ for every $\beta \in \mathbb{N}_{0}^{s}$.

Moreover if $u \in \mathcal{E}_{(\mathfrak{M})}(\Omega)^{\prime}$, is such that $|\langle u, \cdot\rangle| \leq C\|\cdot\|_{K_{0}, h_{0}, j_{0}}$ on $\mathcal{E}_{(\mathfrak{M})}(\Omega)$, then, for any continuous semi-norm $\|\cdot\|_{K^{\prime}, h, j}$ on $\mathcal{E}_{\left(\mathfrak{M}^{\prime}\right)}\left(\Omega^{\prime}\right)$ with $0<h<h_{0}$ and $j>j_{0}$ and every $\beta \in \mathbb{N}_{0}^{s}$, we get

$$
\sup _{y \in K^{\prime}}\left|\mathrm{D}^{\beta}\langle u, f(\cdot, y)\rangle\right| \leq C \sup _{\alpha \in \mathbb{N}_{0}^{r}} \frac{\left\|\mathrm{D}^{(\alpha, \beta)} f\right\|_{K_{0} \times K^{\prime}}}{h_{0}^{|\alpha|} M_{j_{0},|\alpha|}} \leq C h^{|\beta|} M_{j,|\beta|}^{\prime}\|f\|_{K_{0} \times K^{\prime}, h, j} .
$$

Hence $\langle u, f(\cdot, y)\rangle$ belongs to $\mathcal{E}_{\left(\mathfrak{M}^{\prime}\right)}\left(\Omega^{\prime}\right)$ and we have

$$
\|\Lambda f\|_{K^{\prime}, h, j} \leq C\|f\|_{K_{0} \times K^{\prime}, h, j}, \quad \forall f \in \mathcal{E}_{\left(\mathfrak{M} \times \mathfrak{M}^{\prime}\right)}\left(\Omega \times \Omega^{\prime}\right) .
$$

Proposition 15 If $\mathfrak{M}^{\prime}$ is regular, the bilinear map

$$
\Delta: \mathcal{E}_{\left(\mathfrak{M} \times \mathfrak{M}^{\prime}\right)}\left(\Omega \times \Omega^{\prime}\right) \times \mathcal{E}_{(\mathfrak{M})}(\Omega)^{\prime} \rightarrow \mathcal{E}_{\left(\mathfrak{M}^{\prime}\right)}\left(\Omega^{\prime}\right) ; \quad(f, u) \mapsto\langle u, f(\cdot, y)\rangle
$$

is hypocontinuous.

ProOF. By [5, III.5.2] and part b) of the previous Proposition, we only need to prove that, for every $f \in \mathcal{E}_{\left(\mathfrak{M} \times \mathfrak{M}^{\prime}\right)}\left(\Omega \times \Omega^{\prime}\right)$, the linear map

$$
\Delta_{f}: \mathcal{E}_{(\mathfrak{M})}(\Omega)^{\prime} \rightarrow \mathcal{E}_{\left(\mathfrak{M}^{\prime}\right)}\left(\Omega^{\prime}\right) ; \quad u \mapsto\langle u, f(\cdot, y)\rangle
$$

is continuous. This is direct since for every continuous semi-norm $\|\cdot\|_{K^{\prime}, h, j}$ on $\mathcal{E}_{\left(\mathfrak{M}^{\prime}\right)}\left(\Omega^{\prime}\right)$ and every $u \in$ $\mathcal{E}_{(\mathfrak{M})}(\Omega)^{\prime}$, we have

$$
\|\langle u, f(\cdot, y)\rangle\|_{K^{\prime}, h, j}=\sup _{g \in B}|\langle u, g\rangle|
$$

with $B=\left\{\mathrm{D}^{(0, \beta)} f(\cdot, y) /\left(h^{|\beta|} M_{j,|\beta|}^{\prime}\right): \beta \in \mathbb{N}_{0}^{s}, y \in K^{\prime}\right\}$, a bounded subset of $\mathcal{E}_{(\mathfrak{M})}(\Omega)$ indeed. 
Proposition 16 a) For every $\varphi \in \mathcal{D}_{\left(\mathfrak{M} \times \mathfrak{M}^{\prime}\right)}\left(\Omega \times \Omega^{\prime}\right)$ and $y \in \Omega^{\prime}$, the function $\varphi(\cdot, y)$ belongs to $\mathcal{D}_{(\mathfrak{M})}(\Omega)$.

b) If $\mathfrak{M}^{\prime}$ is regular, then, for every $\beta \in \mathbb{N}_{0}^{s}$, the map $D^{(0, \beta)}$ is a continuous linear map from the space $\mathcal{D}_{\left(\mathfrak{M} \times \mathfrak{M}^{\prime}\right)}\left(\Omega \times \Omega^{\prime}\right)$ into itself.

c) If $\mathfrak{M}^{\prime}$ is regular, then, for every $\varphi \in \mathcal{D}_{\left(\mathfrak{M} \times \mathfrak{M}^{\prime}\right)}\left(\Omega \times \Omega^{\prime}\right)$, the function

$$
g: \Omega^{\prime} \rightarrow \mathcal{D}_{(\mathfrak{M})}(\Omega) ; \quad y \mapsto \varphi(\cdot, y)
$$

is a $\mathcal{C}^{\infty}$-function and, for every $\beta \in \mathbb{N}_{0}^{s}$, one has $D^{\beta} g(y)=D^{(0, \beta)} f(\cdot, y)$ for every $y \in \Omega^{\prime}$.

d) Let $\mathfrak{M}^{\prime}$ be regular. If u belongs to $\mathcal{D}_{(\mathfrak{M})}(\Omega)^{\prime}$, then

(i) for every $\varphi \in \mathcal{D}_{\left(\mathfrak{M} \times \mathfrak{M}^{\prime}\right)}\left(\Omega \times \Omega^{\prime}\right),\langle u, \varphi(\cdot, y)\rangle$ belongs to $\mathcal{D}_{\left(\mathfrak{M}^{\prime}\right)}\left(\Omega^{\prime}\right)$ and one has $D^{\beta}\langle u, \varphi(\cdot, y)\rangle=$ $\left\langle u, D^{(0, \beta)} \varphi(\cdot, y)\right\rangle$ for every $\beta \in \mathbb{N}_{0}^{s}$;

(ii) the linear map

$$
\Gamma: \mathcal{D}_{\left(\mathfrak{M} \times \mathfrak{M}^{\prime}\right)}\left(\Omega \times \Omega^{\prime}\right) \rightarrow \mathcal{D}_{\left(\mathfrak{M}^{\prime}\right)}\left(\Omega^{\prime}\right) ; \quad \varphi \mapsto \Gamma \varphi(y)=\langle u, \varphi(\cdot, y)\rangle
$$

is continuous.

e) If $\mathfrak{M}^{\prime}$ is regular, the bilinear map

$$
\Delta: \mathcal{D}_{\left(\mathfrak{M} \times \mathfrak{M}^{\prime}\right)}\left(\Omega \times \Omega^{\prime}\right) \times \mathcal{D}_{(\mathfrak{M})}(\Omega)^{\prime} \rightarrow \mathcal{D}_{\left(\mathfrak{M}^{\prime}\right)}\left(\Omega^{\prime}\right) ; \quad(\varphi, u) \mapsto\langle u, \varphi(\cdot, y)\rangle
$$

is hypocontinuous.

PROOF. In all cases, $\varphi$ belongs to $\mathcal{E}_{\left(\mathfrak{M} \times \mathfrak{M}^{\prime}\right)}\left(\Omega \times \Omega^{\prime}\right)$ and we may apply the previous results.

a) and c) are then clear.

b) In fact, the proof of the corresponding result for $\mathcal{E}_{\left(\mathfrak{M} \times \mathfrak{M}^{\prime}\right)}\left(\Omega \times \Omega^{\prime}\right)$ establishes that the linear map $\mathrm{D}^{(0, \beta)}$ is continuous from $\mathcal{D}_{\left(\mathfrak{M} \times \mathfrak{M}^{\prime}\right)}(K)$ into itself for any compact subset $K$ of $\Omega \times \Omega^{\prime}$.

d) The part (i) is clear. For the part (ii), we note that the restriction of the map $\Lambda$ to $\mathcal{D}_{\left(\mathfrak{M} \times \mathfrak{M}^{\prime}\right)}\left(\Omega \times \Omega^{\prime}\right)$ is of course continuous from $\mathcal{D}_{\left(\mathfrak{M} \times \mathfrak{M}^{\prime}\right)}\left(\Omega \times \Omega^{\prime}\right)$ into $\mathcal{E}_{\left(\mathfrak{M}^{\prime}\right)}\left(\Omega^{\prime}\right)$ and coincides with $\Gamma$. So $\Gamma$ is a linear map with closed graph from an ultrabornological space into a (LF)-space hence is continuous.

e) Here again by [5, III.5.2], we only need to prove that, for every element $\varphi$ of $\mathcal{D}_{\left(\mathfrak{M} \times \mathfrak{M}^{\prime}\right)}\left(\Omega \times \Omega^{\prime}\right)$, the linear map

$$
\Delta_{\varphi}: \mathcal{D}_{(\mathfrak{M})}(\Omega)^{\prime} \rightarrow \mathcal{D}_{\left(\mathfrak{M}^{\prime}\right)}\left(\Omega^{\prime}\right) ; \quad u \mapsto\langle u, \varphi(\cdot, y)\rangle
$$

is continuous. As there are compact subsets $K$ of $\Omega$ and $K^{\prime}$ of $\Omega^{\prime}$ such that $\operatorname{supp}(\varphi) \subset K \times K^{\prime}$, we obtain that the map $\Delta_{\varphi}: \mathcal{D}_{(\mathfrak{M})}(\Omega)^{\prime} \rightarrow \mathcal{D}_{\left(\mathfrak{M}^{\prime}\right)}\left(K^{\prime}\right)$ is continuous since, for every continuous semi-norm $\|\cdot\|_{K^{\prime}, h, j}$ on $\mathcal{D}_{\left(\mathfrak{M}{ }^{\prime}\right)}\left(K^{\prime}\right)$ and every $u \in \mathcal{D}_{(\mathfrak{M})}(\Omega)^{\prime}$, we have

$$
\|\langle u, \varphi(\cdot, y)\rangle\|_{K^{\prime}, h, j} \leq \sup _{g \in B}|\langle u, g\rangle|
$$

with $B=\left\{\mathrm{D}^{(0, \beta)} \varphi(\cdot, y) /\left(h^{|\beta|} M_{j,|\beta|}^{\prime}: \beta \in \mathbb{N}_{0}^{s}, y \in K^{\prime}\right\}\right.$, a bounded subset of $\mathcal{D}_{(\mathfrak{M})}(K)$ hence of $\mathcal{D}_{(\mathfrak{M})}(\Omega)$.

Remark 1 Of course, instead of fixing y in $\Omega^{\prime}$, we may fix $x \in \Omega$ and, up to having $\mathfrak{M}$ regular instead of $\mathfrak{M}^{\prime}$ when necessary, we get analogous results.

\section{The maps $[u, v),(u, v]$ and $u \otimes v$}

Definition 6 Let $u$ and $v$ be continuous linear functionals on $\mathcal{D}_{(\mathfrak{M})}(\Omega)$ and $\mathcal{D}_{\left(\mathfrak{M}^{\prime}\right)}\left(\Omega^{\prime}\right)$ respectively. If $\mathfrak{M}^{\prime}$ is regular, the Proposition 16 tells us that

$$
[u, v): \mathcal{D}_{\left(\mathfrak{M} \times \mathfrak{M}^{\prime}\right)}\left(\Omega \times \Omega^{\prime}\right) \rightarrow \mathbb{C} ; \quad \varphi \mapsto\langle v,\langle u, \varphi(\cdot, y)\rangle\rangle
$$


is a continuous linear functional.

If moreover $u$ and $v$ have compact supports, they have continuous linear extension on $\mathcal{E}_{(\mathfrak{M})}(\Omega)$ and $\mathcal{E}_{\left(\mathfrak{M}^{\prime}\right)}\left(\Omega^{\prime}\right)$ respectively. Therefore by the Proposition $14,[u, v)$ has a continuous linear extension on $\mathcal{E}_{\left(\mathfrak{M} \times \mathfrak{M}^{\prime}\right)}\left(\Omega \times \Omega^{\prime}\right)$ hence has a compact support.

Proposition 17 If $\mathfrak{M}^{\prime}$ is regular, $u \in \mathcal{D}_{(\mathfrak{M})}(\Omega)^{\prime}$ and $v \in \mathcal{D}_{\left(\mathfrak{M}^{\prime}\right)}(\Omega)^{\prime}$, then

(a) $D^{\beta}[u, v)=\left[u, D^{\beta} v\right)$ for every $\beta \in \mathbb{N}_{0}^{s}$;

(b) $(f \otimes g)[u, v)=[f u, g v)$ for every $f \in \mathcal{E}_{(\mathfrak{M})}(\Omega)$ and $g \in \mathcal{E}_{\left(\mathfrak{M}^{\prime}\right)}\left(\Omega^{\prime}\right)$.

PROOF. This is clear since these continuous linear functionals coincide on $\mathcal{D}_{(\mathfrak{M})}(\Omega) \otimes \mathcal{D}_{\left(\mathfrak{M}^{\prime}\right)}\left(\Omega^{\prime}\right)$, a dense subspace of $\mathcal{D}_{\left(\mathfrak{M} \times \mathfrak{M}^{\prime}\right)}\left(\Omega \times \Omega^{\prime}\right)$.

Proposition 18 If $\mathfrak{M}^{\prime}$ is regular, the bilinear map

$$
\mathcal{B}: \mathcal{D}_{(\mathfrak{M})}(\Omega)^{\prime} \times \mathcal{D}_{\left(\mathfrak{M}^{\prime}\right)}\left(\Omega^{\prime}\right)^{\prime} \rightarrow \mathcal{D}_{\left(\mathfrak{M} \times \mathfrak{M}^{\prime}\right)}\left(\Omega \times \Omega^{\prime}\right)^{\prime} ; \quad(u, v) \mapsto[u, v)
$$

is hypocontinuous.

PROOF. As $\mathcal{D}_{(\mathfrak{M})}(\Omega)$ and $\mathcal{D}_{\left(\mathfrak{M}^{\prime}\right)}\left(\Omega^{\prime}\right)$ are (LFS)-spaces, their duals are ultrabornological. Therefore by [5, III.5.2], we only need to prove that $\mathcal{B}$ is separately continuous.

On one hand, if $u$ belongs to $\mathcal{D}_{(\mathfrak{M})}(\Omega)^{\prime}$, we know by part d(ii) of Proposition 16 that $\Gamma$ is a continuous linear map. Therefore its transpose ${ }^{t} \Gamma$ from $\mathcal{D}_{\left(\mathfrak{M}^{\prime}\right)}\left(\Omega^{\prime}\right)^{\prime}$ into $\mathcal{D}_{\left(\mathfrak{M} \times \mathfrak{M}^{\prime}\right)}\left(\Omega \times \Omega^{\prime}\right)^{\prime}$ is continuous with

$$
\left\langle{ }^{t} \Gamma v, \varphi\right\rangle=\langle v, \Gamma \varphi\rangle=\langle v,\langle u, \varphi(\cdot, y)\rangle\rangle
$$

for every $\varphi \in \mathcal{D}_{\left(\mathfrak{M} \times \mathfrak{M}^{\prime}\right)}\left(\Omega \times \Omega^{\prime}\right)$, i.e. ${ }^{t} \Gamma v=[u, v)$.

On the other hand, let $v$ be an element of $\mathcal{D}_{\left(\mathfrak{M}^{\prime}\right)}\left(\Omega^{\prime}\right)^{\prime}$; of course $V=\left\{\psi \in \mathcal{D}_{\left(\mathfrak{M}^{\prime}\right)}\left(\Omega^{\prime}\right):|\langle v, \psi\rangle| \leq 1\right\}$, a 0-neighbourhood in $\mathcal{D}_{\left(\mathfrak{M}^{\prime}\right)}\left(\Omega^{\prime}\right)$. Now let $W$ be any 0-neighbourhood in $\mathcal{D}_{\left(\mathfrak{M} \times \mathfrak{M}^{\prime}\right)}\left(\Omega \times \Omega^{\prime}\right)^{\prime}$. There is then a bounded subset $A$ of $\mathcal{D}_{\left(\mathfrak{M} \times \mathfrak{M}^{\prime}\right)}\left(\Omega \times \Omega^{\prime}\right)$ such that $A^{\circ} \subset W$. As the bilinear map $\Delta$ of part e) of Proposition 16 is hypocontinuous, there is a 0-neighbourhood $U$ in $\mathcal{D}_{(\mathfrak{M})}(\Omega)^{\prime}$ such that $\Delta(A \times U) \subset V$, i.e. such that $B(\cdot, v) U \subset A^{\circ} \subset W$.

Proposition 19 If $\mathfrak{M}^{\prime}$ is regular, the bilinear map

$$
\mathcal{B}: \mathcal{E}_{(\mathfrak{M})}(\Omega)^{\prime} \times \mathcal{E}_{\left(\mathfrak{M}^{\prime}\right)}\left(\Omega^{\prime}\right)^{\prime} \rightarrow \mathcal{E}_{\left(\mathfrak{M} \times \mathfrak{M}^{\prime}\right)}\left(\Omega \times \Omega^{\prime}\right)^{\prime} ; \quad(u, v) \mapsto[u, v)
$$

is continuous.

PROOF. As $\mathcal{E}_{(\mathfrak{M})}(\Omega)$ and $\mathcal{E}_{\left(\mathfrak{M}^{\prime}\right)}\left(\Omega^{\prime}\right)$ are (FS)-spaces, it suffices to prove that $\mathcal{B}$ is separately continuous.

For this purpose, one has just to proceed as in the previous proof replacing on one hand part d(ii) of Proposition 16 by part b) of Proposition 14 and on the other hand part e) of Proposition 16 by Proposition 15.

Remark 2 If $\mathfrak{M}$ is regular, one gets similar results for $(u, v]$ defined as follows

$$
(u, v]: \mathcal{D}_{\left(\mathfrak{M} \times \mathfrak{M}^{\prime}\right)}\left(\Omega \times \Omega^{\prime}\right) \rightarrow \mathbb{C} ; \quad \varphi \mapsto\langle u,\langle v, \varphi(x, \cdot\rangle\rangle
$$

for every $u \in \mathcal{D}_{(\mathfrak{M})}(\Omega)^{\prime}$ and $v \in \mathcal{D}_{\left(\mathfrak{M}^{\prime}\right)}\left(\Omega^{\prime}\right)^{\prime}$.

Proposition 20 If $\mathfrak{M}$ and $\mathfrak{M}^{\prime}$ are regular, then we have $[u, v)=(u, v]$ for every $u \in \mathcal{D}_{(\mathfrak{M})}(\Omega)^{\prime}$ and $v \in \mathcal{D}_{\left(\mathfrak{M}^{\prime}\right)}\left(\Omega^{\prime}\right)^{\prime}$. 
PROOF. Indeed the continuous linear functionals $[u, v)$ and $(u, v]$ on the space $\mathcal{D}_{\left(\mathfrak{M} \times \mathfrak{M}^{\prime}\right)}\left(\Omega \times \Omega^{\prime}\right)$ coincide on the dense linear subspace $\mathcal{D}_{(\mathfrak{M})}(\Omega) \otimes \mathcal{D}_{\left(\mathfrak{M}^{\prime}\right)}\left(\Omega^{\prime}\right)$.

Definition 7 If $\mathfrak{M}$ and $\mathfrak{M}^{\prime}$ are regular, the previous result allows us to define the tensor product $u \otimes v$ of $u \in \mathcal{D}_{(\mathfrak{M})}(\Omega)^{\prime}$ and $v \in \mathcal{D}_{\left(\mathfrak{M}^{\prime}\right)}\left(\Omega^{\prime}\right)^{\prime}$ by $u \otimes v=[u, v)=(u, v]$.

Proposition 21 If $\mathfrak{M}$ and $\mathfrak{M}^{\prime}$ are regular, then, for every $u \in \mathcal{D}_{(\mathfrak{M})}(\Omega)^{\prime}$ and $v \in \mathcal{D}_{\left(\mathfrak{M}^{\prime}\right)}\left(\Omega^{\prime}\right)^{\prime}$, we have

(a) $\operatorname{supp}(u \otimes v)=\operatorname{supp}(u) \times \operatorname{supp}(v)$;

(b) $D^{(\alpha, \beta)}(u \otimes v)=\left(D^{\alpha} u\right) \otimes\left(D^{\beta} v\right)$ for every $\alpha \in \mathbb{N}_{0}^{r}$ and $\beta \in \mathbb{N}_{0}^{s}$.

Acknowledgement. The second author was partially supported by MEC and FEDER Project MTM 2005-08210.

\section{References}

[1] Beaugendre, P., (2001). Extension de jets dans des intersections de classes non quasi-analytiques, Ann. Polon. Math., LXXVI, 214-243.

[2] Beaugendre, P., (2002). Intersection de classes non quasi-analytiques, Thèse de doctorat, Université de Paris XI, UFR Scientifique d'Orsay, 2404, 84 pp.

[3] Chaumat, J. And Chollet, A.-M., (1998). Propriétés de l'intersection des classes de Gevrey et de certaines autres classes, Bull. Sci. math., 122, 455-485.

[4] JARCHOW, H., (1981). Locally convex spaces, B. G. teubner Mathematische Leitfäden.

[5] SCHAEFER, H., (1971). Topological vector spaces, Springer Graduate Texts in Mathematics, 3.

[6] Schmets, J. And Valdivia, M., (2005/2006). Extension properties in intersections of non quasi-analytic classes, Note Mat., 25, 159-185.

[7] Schmets, J. And Valdivia, M., (2005). Explicit extension maps in intersections of non quasi-analytic classes, Ann. Polon. Math., 86, 227-243.

[8] Schmets, J. AND VAldivia, M., About some non quasi-analytic classes, submitted for publication, 24 pp.

[9] Schmets, J. AND VAldivia, M., Intersections of non quasi-analytic classes, submitted, 15 pp.

[10] Schmets, J. AND VAldivia, M., Tensor product characterization of mixed intersections of non quasi-analytic classes and kernel theorems, preprint, $10 \mathrm{pp}$.

\author{
Jean Schmets \\ Institut de Mathématique \\ Université de Liège \\ Sart Tilman Bât. B 37 \\ B-4000 LIEGE 1 \\ BELGIUM \\ j.schmetseulg.ac.be
}

\author{
Manuel Valdivia \\ Facultad de Matemáticas \\ Universidad de Valencia \\ Dr. Moliner 50 \\ E-46100 BURJASOT (Valencia) \\ SPAIN
}

